letter to the editor

\title{
Authors' reply to Finsterer and Aliyev
}

Dear Sir,

We recently described a 70-year-old woman who developed Takotsubo syndrome (TTS) after a left middle cerebral artery stroke (Bersano et al., 2014). In their letter commenting on our paper, published in the present issue, Finsterer and Aliyev (2014) raise some doubts regarding the role of TTS as a complication rather than the cause of the initial stroke. Our patient presented at the emergency unit of our hospital with an acute left temporoparietal infarction. She was treated with rTPA 120 minutes from stroke onset with right hemiparesis improvement but residual aphasia (reduction of NIHSS score from 12 to 4). On admission, the patient did not present clinical signs or symptoms (e.g. dyspnea or leg edema) or ECG signs of cardiac failure and/or coronary artery disease (CAD). Continuous stroke unit ECG monitoring was maintained for 72 hours, but it did not show any ST segment alterations, QT prolongation or T-wave changes. Also brain natriuretic peptide and cardiac acute phase enzymatic profiles were negative. During the hospitalization, the patient developed a new small right cortical and subcortical temporoparietal stroke, documented by a new CT scan and causing a left arm motor and sensory deficit. Concomitantly an ST segment elevation and increased BNP levels were found and an intracardiac ballooning thrombus consistent with TTS was detected on transthoracic echocardiography (TEE). Although an acute-phase TEE was not performed, the clinical history and the laboratory data reliably suggest that TTS was a consequence rather than the cause of the first stroke, possibly favored by the insular site of the cerebral infarction. To ascertain a possible underlying CAD a CT angiography was performed. Although traditional coronary angiography is the gold standard for CAD diagnosis, recent trials showed that CT angiography, which is a noninvasive technique, is able to provide a similar diagnostic accuracy for the diagnosis of hemodynamically significant CAD (Nørgaard et al., 2014). Given the relative risk due to the small size of the two strokes, anticoagulation therapy was started relatively early. Although current guidelines and analysis of the largest randomized trial do not recommend the use of anticoagulants for the management of acute stroke (Jauch et al., 2013; Whiteley et al., 2013), the value of this approach has not been established for any patient group and uncertainty remains regarding the role of anticoagulants in patients with uncommon conditions (Eikelboom et al., 2013) causing acute brain ischemia, such as TTS.

However, as stated by Finsterer et al., our case highlights the possible causative role of TTS and supports the need for awareness among neurologists and cardiologists concerning the diagnosis and treatment of TTS. In fact TTS is an increasingly recognized phenomenon associated with CNS disease that has to be considered as a possible complication of subarachnoidal bleeding, epilepsy, and ischemic stroke through catecholamine release due to these stress conditions. TTS must be promptly recognized and treated since it may be further complicated by arrhythmias, thrombus formation, or heart failure, which may secondarily lead to stroke (Finsterer et al., 2014).

Anna Bersano, MD, PhDa*, Claudio Bassetti, $M D^{b}$

${ }^{a}$ Cerebrovascular Unit, Fondazione IRCCS Istituto Neurologico Carlo Besta, Milan, Italy ${ }^{b}$ Neurology Department, Inselspital, Bern University Hospital, Bern, Switzerland

*Correspondence to: anna.bersano@gmail.com

\section{References}

Bersano A, Melchiorre P, Moschwitis G, et al (2014). Tako-tsubo syndrome as a consequence and cause of stroke. Funct Neurol 29:135-137. Eikelboom JW, Hart RG (2013). Anticoagulant therapy in acute brain ischaemia. Lancet Neurol 12:526-527.

Finsterer J, Aliyev R (2014). Takotsubo syndrome: consequence or cause of ischemic stroke. Funct Neurol 29:282.

Finsterer J, Wahbi K (2014). CNS disease triggering Takotsubo stress cardiomyopathy. Int J Cardiol 177:322-329.

Jauch EC, Saver JL, Adams HP Jr, et al (2013). Guidelines for the early management of patients with acute ischemic stroke: a guideline for healthcare professionals from the American Heart Association/American Stroke Association. Stroke 44:870-947.

Nørgaard BL, Leipsic J, Gaur S,et al (2014). Diagnostic performance of noninvasive fractional flow reserve derived from coronary computed tomography angiography in suspected coronary artery disease: the NXT trial (Analysis of Coronary Blood Flow Using CT Angiography: Next Steps). J Am Coll Cardiol 63:1145-1155.

Whiteley WN, Adams HP Jr, Bath PM, et al (2013). Targeted use of heparin, heparinoids, or low-molecular-weight heparin to improve outcome after acute ischaemic stroke: anindividual patient data meta-analysis of randomised controlled trials. Lancet Neurol 12:539-545. 